\title{
Musculoskeletal Disorder among 52,261 Chinese Restaurant Cooks Cohort: Result from the National Health Insurance Data
}

\author{
Huei-Sheng Shiue ${ }^{1,2}$, Chih-Wei Lu' ${ }^{1}$, Chiou-Jong Chen ${ }^{3}$, Tung-Shen Shin ${ }^{3,4}$, \\ Shiao-Chi Wu ${ }^{5}$, Chun-Yuh YANG ${ }^{6}$, Ya-Hui Y $\mathrm{ANG}^{1}$ and Trong-Neng Wu${ }^{4,7}$ \\ ${ }^{1}$ Graduate Institute of Occupational Safety and Health, Kaohsiung Medical University, ${ }^{2}$ Department of Physical \\ Medicine and Rehabilitation, E-Da Hospital/I-Shou University, ${ }^{3}$ Institute of Occupational Safety and Health, Labor \\ Affairs Council, ${ }^{4}$ Institute of Environmental Health, College of Public Health, China Medical University and \\ Hospital, ${ }^{5}$ Institute of Health and Welfare Policy, National Yang Ming University, ${ }^{6}$ Faculty of Public Health, \\ Kaohsiung Medical University and ${ }^{7}$ Institute of Environmental Health, National Yang Ming University, Taiwan
}

\begin{abstract}
Musculoskeletal Disorder among 52,261 Chinese Restaurant Cooks Cohort: Result from the National Health Insurance Data: Huei-Sheng SHIUE, et al. Graduate Institute of Occupational Safety and Health, Kaohsiung Medical University, Taiwan-To investigate the incidence of musculoskeletal disorders (MSDs) and the particular MSDs to which Chinese restaurant cooks are most at risk in Taiwan, National Health Insurance Data from1998 to 2002 were used to identify MSDs for study populations, including 52,261 certified cooks and 209,044 references matched for age and sex. The annual incidence of MSDs was around $25 \%$ and $20 \%$ for cooks and references, respectively, and the cook-to-reference risk ratios ranged from 1.29 to $1.35(p<0.001)$. The most frequently affected body part was the low back, but the epicondyle was at higher risk $(\mathrm{OR}>2)$ than other sites for the cooks. Cooks are at higher risk of having MSDs complaints and MSDs related to the elbow show the highest risk.
\end{abstract}

(J Occup Health 2008; 50: 163-168)

Key words: Chinese restaurant cooks, Health insurance, Musculoskeletal disorder (MSD), Occupational disease, Incidence

Musculoskeletal disorders (MSDs) are common complaints in industry, particularly among workers with intensive manual labor, as a leading cause of work related illness. In many countries, MSDs are also the leading cause of work-related illness. The Health and Safety

Received Jul 9, 2007; Accepted Dec 7, 2007

Correspondence to: T. $-N$. Wu, Institute of Environmental Health, College of Public Health, China Medical University and Hospital, 91 Hseuh-Shih Road, Taichung 404, Taiwan

(e-mail: tnwu@mail.cmu.edu.tw)
Executive of the U.K. has reported that MSDs are the most common occupational illness in Great Britain, affecting 1.0 million people anually with a cost to society of $£ 5.7$ billion $^{1)}$. In the U.S., low back pain accounts for one fourth of compensation claims and one third of compensation costs ${ }^{2}$. Gou et al. found that $37 \%$ workers in Taiwan had subjective symptoms of $\mathrm{MSDs}^{3}$. Numerous reports on MSDs have been published but few of them have concerned cooks.

Repetitive manual work, lifting and forceful movements, awkward posture and efforts are well-known risk factors contributing to MSDs ${ }^{4-8)}$. A cook's work is characterized by long standing hours, constant leaning forward of the body, and repetitive motion in the upper limbs ${ }^{9,10)}$. Because of the high body strain associated with preparing raw materials and cuisine, cooks and restaurant workers are at high risk of MSDs ${ }^{11-13)}$. Most studies of MSDs have considered subjective health complaints obtained with questionnaire survey. Some of these complaints might be minor without the need for medical attention. Therefore, workers with MSDs seeking medical care deserve better understanding. The purpose of this study was to investigate the incidence and risk of MSDs and the particular MSDs to which Chinese restaurant cooks are most at risk in Taiwan using health insurance data.

\section{Methods}

Study population

The study population included 65,535 certified cooks who had registered with the Bureau of Employment and Vocational Training (BEVT) in Taiwan and worked in the business of Chinese cuisine. Individuals with duplicated files, incomplete information, and/or aged less than $18 \mathrm{yr}$ in 1998 were excluded from the data analyses. 


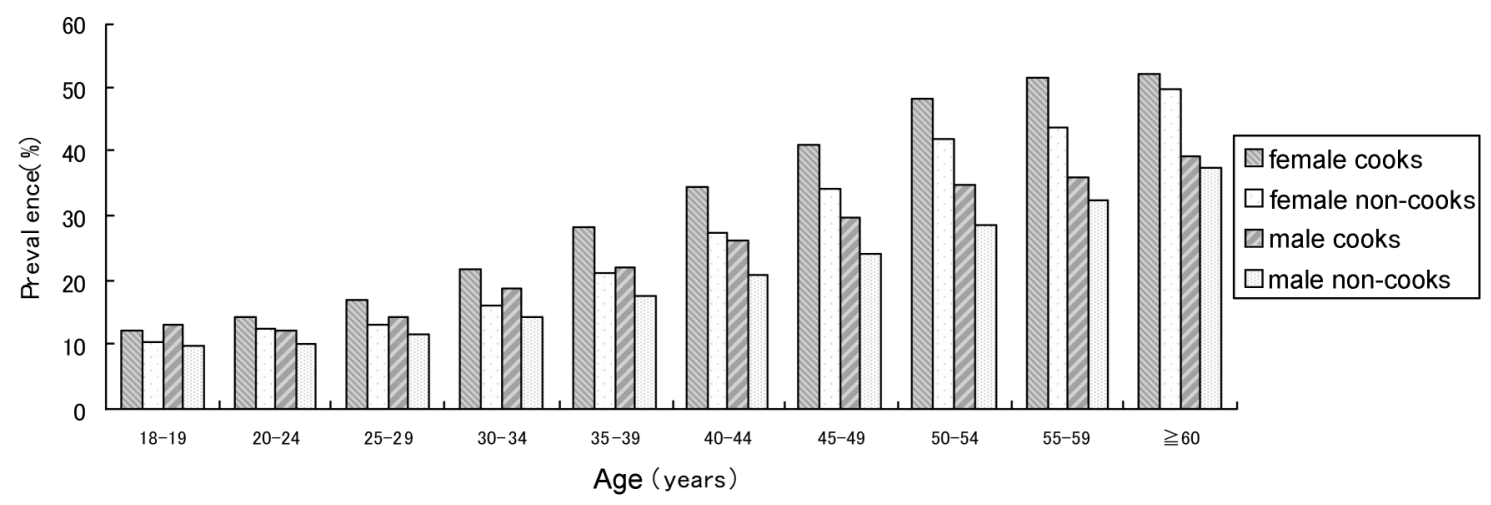

Fig. 1. Five-year age and gender-specific average incidence of musculoskeletal disorders between cooks and references (non-cooks).

Valid cooks were 22,445 men and 29,816 women in 2003. The reference subjects were from the general population selected from the 2003 reimbursement claims data of the National Health Insurance (NHI) of Taiwan. For each cook, four reference subjects were identified randomly, and matched for sex and age (within one year). Implemented in 1995, this insurance program covered $96.2 \%$ of population in $2003^{14}$. The National Health Insurance Bureau provided electronic data with patients' sex, birthday, the classification code of the disease diagnosed, data of health services received, and the clinic or hospital code. Thus, 209,044 non-cooks were identified from the NHI files as reference subjects for this study. Using the national registration identification numbers of cooks obtained from BEVT, they were linked to the insurance data. Information associated with these cooks extracted from the NHI data were merged with the data of the reference subjects to establish a data set with scrambled patient identification numbers to protect anonymity.

\section{Definitions}

Cases of musculoskeletal disorders were identified from the NHI data for the years from 1998 to 2002 using the 9th revision of the International Classification of Diseases (ICD), for both the certified Chinese restaurant cooks and the reference group. Musculoskeletal disorders (MSDs) were defined as patients diagnosed with either an ICD-9 code between 710 to 739 or A-code (abridged code) between 431 to 439 . The A codes were converted into ICD-9 for data analysis. In this study, we were concerned about the leading causes of work-related and repetitive motioning disorders, so we excluded tumor (ICD-code 170, 171, and 213) or trauma (injury or poisoning, ICD-9-CM code between 800 to 999).

The relationship between repeated motion in the upper limbs and regional tendinitis had been well documented ${ }^{4-8)}$. Thus, we chose tendonitis of the shoulder, elbow, and wrist to investigate the occupational risk of the cooks. As well as repetitive motion, the cooks often bend or twist their wrists and this has also been reported as a risk factor of carpal tunnel syndrome, so carpal tunnel syndrome was also included as an occupational disease. Prolonged standing and lifting of heavy objects contributes to low back pain, so disc herniation of the lumbar spine and lumbago were also chosen. Therefore, the incidence of common work-related MSDs were compared in this study, including displacement of the thoracic or lumbar intervertebral disc without myelopathy (ICD-9 code 722.1), lumbago (ICD9 code 724.2), rotator cuff syndrome of the shoulder (ICD9 code 726.1), medial epicondylitis (ICD-9 code 726.31), lateral epicondylitis (ICD-9 code 726.32), trigger finger (ICD-9 code 727.03), radial styloid tenosynovitis (ICD9 code 727.04), and carpal tunnel syndrome (ICD-9 code 354.0).

\section{Statistical methods}

First we calculated the overall MSD complaints for the two study groups in 1998-2002. The annual incidence was the number of new cases that developed the MSDs divided by the size of the population at risk in each year; the 5-yr cumulative incidence was the number of new cases that developed the MSDs divided by the size of the population at risk from 1998 to 2002. The annual incidence of MSDs was calculated for the study group, with cooks-to-references risk ratios presented as odds ratio (OR) and $95 \%$ confidence interval (CI). The chisquare test was used to obtain the OR and 95\% CI. Data analysis further compared the average age-specific incidences in these five years between the two groups. The site-specific incidences of MSDs for the cooks and references were also compared. In order to identify sexspecific differences in the MSD risk, we also calculated the mean of sex-age-specific incidence for cooks and references (Fig. 1). 
Table 1. Demographic data and five-year cumulative incidence of MSDs in Chinese restaurant cooks and references

\begin{tabular}{llcc}
\hline & & Cooks & References \\
\hline $\mathrm{N}$ & & 52,261 & 209,044 \\
Gender & Female & $29,816(57.1 \%)$ & $119,264(57.1 \%)$ \\
& Male & $22,445(42.9 \%)$ & $89,780(42.9 \%)$ \\
\multirow{2}{*}{ Age $(\mathrm{yr})$} & Female & $35.3 \pm 10.1$ & $35.3 \pm 10.1$ \\
& Male & $32.3 \pm 9.3$ & $32.3 \pm 9.3$ \\
Five-year cumulative incidence of MSDs & a,b,c & $31,110(59.5 \%)$ & $106,369(50.9 \%)$ \\
\hline
\end{tabular}

aMSDs: musculoskeletal disorders. ${ }^{b}$ The five-year cumulative incidence was the number of new cases that developed MSDs divided by the size of the population at risk from 1998 to 2002 . ${ }^{c} p$-value $<0.001$.

Table 2. The annual incidence of musculoskeletal disorders for Chinese restaurant cooks and references, 1998-2002

\begin{tabular}{llcccc}
\hline Year & \multicolumn{2}{c}{ Incidence $(\%)$} & OR $^{\mathrm{b}}$ & $(95 \% \mathrm{CI})^{\mathrm{b}}$ & $p$-value \\
\cline { 2 - 5 } & Cooks & References & & & \\
\hline 1998 & 23.60 & 18.72 & 1.34 & $(1.31-1.37)$ & $<0.001$ \\
1999 & 24.62 & 19.68 & 1.33 & $(1.30-1.36)$ & $<0.001$ \\
2000 & 25.87 & 20.56 & 1.35 & $(1.32-1.38)$ & $<0.001$ \\
2001 & 25.98 & 21.17 & 1.31 & $(1.28-1.34)$ & $<0.001$ \\
2002 & 26.25 & 21.65 & 1.29 & $(1.26-1.32)$ & $<0.001$ \\
\hline
\end{tabular}

aThe annual incidence was the number of new cases that developed MSDs divided by the size of the population at risk in each year. ${ }^{b}$ Chi-square test.

\section{Results}

The sex-distribution ( $57.1 \%$ women and $42.9 \%$ men) and their average ages (35.3 yr in women and $32.3 \mathrm{yr}$ in men) in cooks were the same as those in the reference group (Table 1). The 5-yr cumulative incidence of having had MSD was higher in the cooks than in the references (59.5\% vs. $50.9 \%, p<0.001)$. Table 2 shows that the annual incidence of MSDs for the cooks ranged from $23.60 \%$ to $26.25 \%$ and that for the references ranged from $18.72 \%$ to $21.65 \%$. The cooks-to-references odd ratios in 1998-2002 showed that cooks were 1.29-1.34 more likely than references to receive MSD care.

\section{Age-specific incidence}

There was an increasing trend with age for MSD incidence in both the cooks and the reference group (Table 3 ). The age-specific rate increased from approximately $13.22 \%$ in the $20-24 \mathrm{yr}$ old group to $46.92 \%$ in the $60 \mathrm{yr}$ old and above group for cooks. The age-specific incidence in the cooks was consistently greater than that in the references. The cooks-to-references odds ratio peaked in the 35-39 yr old groups (OR 1.42, 95\% CI 1.39-1.46).
Sex-age-specific comparison

Figure 1 shows the sex- and age-specific incidence of having MSDs for cooks and references. In both men and women, the cooks had a higher risk of acquiring MSDs than the male references. In general, starting with the 20-24 yr old group, female cooks had the highest incidence in almost every age group, followed by the female reference groups. The incidence increased as age increased. The incidence of MSDs in female cooks aged $\geq 60 \mathrm{yr}$ was much greater than that in the age group of 20-24 yr, $52.3 \%$ vs. $14.2 \%$.

\section{Incidence of specific sites}

Table 4 shows the work-related MSDs incidence for specific body sites diagnosed in 2001 and 2002. The cooks had higher incidences of complaints for all types of MSD than the references. The incidence of lumbago (ICD code 724.2) was the highest among these disorders in both study groups. The highest OR was found in medial epicondylitis (ICD code 726.31), followed by lateral epicondylitis (ICD code 726.32). The incidences of carpal tunnel syndrome were also higher in cooks than in references with the cooks-to-references OR of 1.76 (95\% CI: 1.5-2.1). 
Table 3. Average five-year age-specific incidence of musculoskeletal disorders among cooks and references from 1998 to 2002

\begin{tabular}{|c|c|c|c|c|c|}
\hline \multirow[t]{2}{*}{ Age (yr) } & \multicolumn{2}{|c|}{$\begin{array}{c}\text { Five-year cumulative } \\
\text { incidence }^{\mathrm{a}}(\%)\end{array}$} & \multirow[t]{2}{*}{$\mathrm{OR}^{\mathrm{b}}$} & \multirow[t]{2}{*}{$95 \% \mathrm{CI}^{\mathrm{b}}$} & \multirow[t]{2}{*}{$p$-value ${ }^{b}$} \\
\hline & Cooks & References & & & \\
\hline $18-19$ & 12.48 & 10.18 & 1.26 & $1.16-1.37$ & $<0.0001$ \\
\hline $20-24$ & 13.22 & 11.33 & 1.19 & $1.15-1.23$ & $<0.0001$ \\
\hline $25-29$ & 15.26 & 12.06 & 1.31 & $1.27-1.36$ & $<0.0001$ \\
\hline $30-34$ & 20.06 & 15.12 & 1.41 & $1.37-1.45$ & $<0.0001$ \\
\hline $35-39$ & 25.78 & 19.65 & 1.42 & $1.39-1.46$ & $<0.0001$ \\
\hline $40-44$ & 31.84 & 25.20 & 1.39 & $1.36-1.42$ & $<0.0001$ \\
\hline $45-49$ & 37.77 & 31.39 & 1.33 & $1.29-1.36$ & $<0.0001$ \\
\hline $50-54$ & 43.99 & 37.71 & 1.30 & $1.25-1.35$ & $<0.0001$ \\
\hline $55-59$ & 46.53 & 40.30 & 1.29 & $1.21-1.37$ & $<0.0001$ \\
\hline$\geq 60$ & 46.92 & 44.57 & 1.10 & $1.00-1.21$ & 0.044 \\
\hline
\end{tabular}

aThe five-year cumulative incidence was the number of new cases that developed MSDs divided

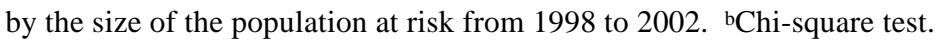

Table 4. Incidence of specific work-related musculoskeletal disorders in cooks and references, and cooks to references risk ratios in 2001 and 2002

\begin{tabular}{|c|c|c|c|c|c|c|c|c|c|}
\hline \multirow{3}{*}{\multicolumn{2}{|c|}{ ICD-9 code }} & \multicolumn{4}{|c|}{2001} & \multicolumn{4}{|c|}{2002} \\
\hline & & \multicolumn{2}{|c|}{ Incidence $(\%)$} & \multirow{2}{*}{$\begin{array}{c}\mathrm{OR}^{\mathrm{b}} \\
(95 \% \mathrm{CI})^{\mathrm{b}}\end{array}$} & \multirow[t]{2}{*}{$p$-value } & \multicolumn{2}{|c|}{ Incidence $(\%)$} & \multirow{2}{*}{$\begin{array}{c}\mathrm{OR}^{\mathrm{b}} \\
(95 \% \mathrm{CI})^{\mathrm{b}}\end{array}$} & \multirow[t]{2}{*}{$p$-value b } \\
\hline & & Cooks & References & & & Cooks & References & & \\
\hline 722.1 & HIVD & 0.66 & 0.48 & $1.38(1.22-1.56)$ & $<0.001$ & 0.70 & 0.49 & $1.43(1.27-1.61)$ & $<0.001$ \\
\hline 724.2 & Lumbago & 4.91 & 4.05 & $1.22(1.17-1.28)$ & $<0.001$ & 4.73 & 3.88 & $1.23(1.17-1.29)$ & $<0.001$ \\
\hline 726.1 & $\begin{array}{l}\text { Rotator cuff syndrome } \\
\text { of shoulder }\end{array}$ & 0.48 & 0.33 & $1.49(1.29-1.72)$ & $<0.001$ & 0.58 & 0.35 & $1.66(1.45-1.90)$ & $<0.001$ \\
\hline 726.31 & Medial epicondylitis & 0.05 & 0.03 & $2.08(1.3$ & & 0.06 & 0.02 & $2.52(1.5$ & $<0.001$ \\
\hline 726.32 & Lateral epicondylitis & 0.43 & 0.21 & $2.10(1.79-2.47)$ & $<0.001$ & 0.48 & 0.24 & $1.96(1.68-2.28)$ & $<0.001$ \\
\hline 727.03 & Trigger finger & 0.21 & 0.11 & $1.92(1.53-2.42)$ & $<0.001$ & 0.23 & 0.13 & $1.75(1.41-2.17)$ & $<0.001$ \\
\hline 727.04 & $\begin{array}{l}\text { Radial styloid } \\
\text { tenosynovitis }\end{array}$ & 0.10 & 0.06 & $1.90(1.37-2.62)$ & $<0.001$ & 0.09 & 0.06 & $1.42(1.01-1.98)$ & 0.042 \\
\hline 354.0 & Carpal tunnel syndrome & e 0.43 & 0.24 & $1.76(1.50-2.06)$ & $<0.001$ & 0.46 & 0.26 & $1.76(1.51-2.05)$ & $<0.001$ \\
\hline
\end{tabular}

${ }^{a}$ The annual incidence was the number of new cases that developed the MSDs divided by the size of the population at risk in each

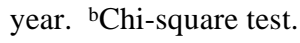

\section{Discussion}

Overall incidence and incidence by disease

To the authors' knowledge, this study is the first to report the physician-diagnosed incidence of MSDs with a large sample for cooks in the business of Chinese cuisine. The strength of this study is that all cooks included in this study were government certified instead of self-reported, therefore it is unlikely to have misclassification of the cooks' status was unlikely. The National Health Insurance in Taiwan is a mandatoryenrollment universal health insurance program with a coverage rate of higher than $96.2 \%^{14)}$. The randomly selected references in this study make the measures of incidence reliable and similar to using the whole population as the denominator. Thus, selection bias was substantially reduced. No previous studies have used national-wide insurance health data to investigate musculoskeletal disorders for cooks. The large sample size allowed the assessment of multiple-site information on specific MSDs with great accuracy and fewer common complaints.

One limitation of this study is that it cannot be sure that all of the registered cooks remained at work at restaurants during this study period. Some of them may have quit the job of cooking during the study period. 
However, the incidence of MSD was only slightly under estimated because of low job turnover among cooks. An other limitation is that the insurance program has not been verified for the ICD codes in the claim files. To reduce the influence of inappropriate records, all ICD-9 codes under the category of musculoskeletal and soft tissue disorders were included. Inappropriate records and misdiagnosis were likely minor and unlikely to be different between the groups. Therefore, underestimate of the relative risk was not likely. We are also concerned that some cooks not registered with the government were selected for the reference group. But, the chance of selecting unregistered cooks from a large general population size (approximately 23 millions) was very minor.

The incidence of MSDs in the study populations was around $25 \%$ annually in average or $59.5 \%$ in the $5-\mathrm{yr}$ observation period, lower than the incidences reported in other studies ${ }^{3,11-13,15)}$. Cooks at hotels in Taiwan have high reported incidences of low back pain (54.7\%), neck pain $(55.6 \%)$, and shoulder pain $(58.9 \%)^{12)}$. A Norwegian study showed that $80 \%$ of hotel foods service employees reported a lifetime experience of MSDs, including 39.3\% of low back pain and $42.4 \%$ of shoulder pain ${ }^{16)}$. Most previous studies have used self-reported subjective symptoms of MSDs, Parkes et al. found that less than half of workers who had suffered from MSDs would visit a doctor ${ }^{15)}$. It is likely a large number of Chinese restaurant cooks did not seek medical assistance for MSDs.

The incidence of lumbago was much higher than other selected MSDs in this survey. It was compatible with most studies that back pain is the most frequently reported

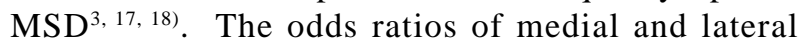
epicondylitis were also significant. The risk for epicondylitis was nearly two times higher in cooks than in the general population. Ono et al. performed physical examinations of 200 female cooks aged from 40 to $59 \mathrm{yr}$, and also found a significantly higher incidence of epicondylitis in cooks $(11.5 \%)$ than in references $(2.5 \%)$ with an adjusted OR of 5.4 ( $95 \%$ CI 2.4 to 11.9$)^{10)}$.

\section{Incidence by age and gender}

An increasing trend with age for MSD incidence was noted in this study. We also examined the interaction of age and sex. The incidence of MSD complaints was higher in women than in men and the gender difference increased with increasing age. Female cooks aged $60 \mathrm{yr}$ and older were approximately three times more likely than young female cooks to seek medical assistance for MSDs. The variation of incidence by age and gender is based on help-seeking behavior for MSD. Old workers were more likely to seek help than younger groups. A Japanese study also found that pains for female kitchen workers increased successively with age. The pain in the axial area, and the upper and lower limbs increased from $22.0 \%$ among workers aged less than $40 \mathrm{yr}$ to $49.0 \%$ among those of $51 \mathrm{yr}$ and older ${ }^{10)}$. Häkkänen et al. followed up 532 trailer assembly workers and found that women have a higher rate of sick leave than men $(3.3 \%$ vs.2.2\%) particularly for neck and shoulder disorders (relative risk $=12.8,95 \%$ CI 3.7-44.5) ${ }^{19}$ ). A recent Norwegian study had a similar finding, that women are at higher risk than men for low back pain $(43.9 \%$ vs. $35.2 \%)$ and shoulder pain $(51.5 \% \text { vs. } 32.0 \%)^{16)}$. In our study, gender difference in the incidence of MSDs was also noted; women were at higher risk of MSDs than men. In the oldest groups, the relative risk between the cooks and the referents reduced. It is likely that workrelated MSDs reduced after work load lessened with age and the incidence of osteoarthritis significantly increased with age (data not shown).

These results demonstrate that Chinese restaurant cooks are at higher risk of having MSDs than the general population. The most frequently affected body part was the low back, but when compared to other populations, upper limbs were at higher risk than the other sites of the body, especially elbows. Ergonomic studies should be launched to innovate upon the working style to reduce MSDs among Chinese restaurant cooks.

Acknowledgment: This study was conducted with support from Institute of Occupational Safety and Health, Labor Affairs Council, Executive Yuan, Taiwan.

\section{References}

1) Health and Safety Executive: Musculoskeletal Disorders. Health and Safety Executive (2006). (online), available from < http://www.hse.gov.uk/msd/ mac/>, (acceded 2007-10-03).

2) Klein BP, Jensen RC and Sanderson LM: Assessment of workers' compensation claims for back strains/ sprains. J Occup Med 26, 443-448 (1984)

3) Guo HR, Chang YC, Yeh WY, Chen CW and Guo YL: Incidence of musculoskeletal disorder among workers in Taiwan: a nationwide study. J Occup Health 46, 2636 (2004)

4) Malchaire J, Cock N and Vergracht S: Review of the factors associated with musculoskeletal problems in epidemiological studies. Int Arch Occup Environ Health 74, 79-90 (2001)

5) Armstrong TJ and Chaffin DB: Carpal tunnel syndrome and selected personal attributes. J Occup Med 21, 481486 (1979)

6) National Institute for Occupational Safety and Health (NIOSH). A critical review of epidemiologic evidence for work-related musculoskeletal disorders of the neck, upper extremity, and low back. Cincinnati: U.S. Department of Health and Human Services, 1997.

7) Latko WA, Armstrong TJ, Franzblau A, Ulin SS, Werner RA and Albers JW: Cross-sectional study of the relationship between repetitive work and the 
incidence of upper limb musculoskeletal disorders. Am J Ind Med 36, 248-259 (1999)

8) de Zwart BC, Frings-Dresen MH and Kilbom A: Gender differences in upper extremity musculoskeletal complaints in the working population. Int Arch Occup Environ Health 74, 21-30 (2001)

9) Messing $K$ and Kilbom A: Standing and very slow walking: foot pain-pressure threshold, subjective pain experience and work activity. Appl Ergon 32, 81-90 (2001)

10) Ono $Y$, Nakamura $R$, Shimaoka $M$, Hiruta $S$, Hattori Y, Ichihara G, Kamijima M and Takeuchi Y: Epicondylitis among cooks in nursery schools. Occup Environ Med 55, 172-179 (1998)

11) Haukka E, Leino-Arjas $P$, Solovieva $S$, Ranta R, Viikari-Juntura E and Riihimaki H: Co-occurrence of musculoskeletal pain among female kitchen workers. Int Arch Occup Environ Health 80, 141-148 (2006)

12) Chyuan JYA, Du CL, Yeh WY and Li CY: A crosssectional study of musculoskeletal isorders in relation to work movement characteristics among hotel foodservice employees in Taiwan. Taiwan J Public Health 21, 140-149 (2002)

13) Chyuan JYA, Ho JH and Sung FC: Risk factors associated with work-related musculoskeletal discomfort among commissary foodservice workers. Taiwan J Public Health 24, 154-161 (2005)

14) Bureau of National Health Insurance: Department of Health, The Executive Yuan, Taiwan. BNHI, 2005. (online), available from <http://www.nhi.gov.tw/ english/index.asp>, (acceded 2006-06-30).

15) Parkes KR, Carnell S, Farmer E. Musculoskeletal disorders, mental health and the work environment. 2005. (online), available from <http://www.hse.gov.uk/ statistics/2002/swi95.pdf>, (acceded 2006-06-30).

16) Ihlebaek $C$, Eriksen $H R$ and Ursin H: Incidence of subjective health complaints (SHC) in Norway. Scand J Public Health 30, 20-29 (2002)

17) Eriksen HR, Svendsrod R, Ursin G and Ursin H: Incidence of subjective health complaints in the Nordic European countries in 1993. Eur J Public Health 8, 294-298 (1998)

18) Jones JR, Hodgson JT, Clegg TA and Elliott RC: Selfreported Work-related Illness in 1995: Results from a Household Survey.1998. (online), available from<http:/ /www.hse.gov.uk/statistics/2002/swi95.pdf>, (acceded 2006-06-30).

19) Häkkänen M, Viikari-Juntura E and Martikainen R: Job experience, work load, and risk of musculoskeletal disorders. Occup Environ Med 58, 129-135 (2001) 